Backenstrass, M. 219

Banzato, C.E.M. 159, 228

Berganza, C.E. 159, 162, 166, 228

Fabrega, H., Jr. 223

First, M.B. 206

Fulford, K.W.M. 171

Harré, R. 185

Jablensky, A. 201

Kirmayer, L.J. 192
Mezzich, J.E. 159, 162, 166, 177, 228

Mundt, C. 219

Phillips, J. 180

Plantikow, T. 215

Pouncey, C. 166

Robert, J.S. 215

Sadler, J.Z. 197

Strauss, J.S. 189

Widiger, T.A. 211

\title{
Subject Index Vol. 38, No. 4, 2005
}

Cognitive tools 185

Complaints, psychotherapy 185

Cultural diversity 192

- evolution 223

Diagnosis, categorical 180, 211

-, clinical 162

-, dimensional 211

-, psychiatric 166

Diagnostic classification 197, 211

- comorbidity 206

- models 177

- process 189

- systems 162

Diagnostics, mental disorders 166

Doctor-patient interaction 192

Ethnography 192

Evolutionary continuum 223

Exclusionary relationship 206

Genetics 215

Genomics 215
Humanities

Idiographic formulation 180

International classifications 162

- -, prototypes 201

Methodological dichotomies 219

Neurosciences 215

Nosology 201, 215

-, psychiatric 166, 180, 192

Positioning theory 185

Psychiatric condition memes 223

- disorders 223

Psychotherapy 219

Robustness 215

Stakeholders 197

Subjective perception 189

Taxonomy 185

Values 197

Values-based practice 171

- - classification 171

Well-being and health 177 